\title{
The Enhancing Effect of Jasmonic Acid on Fragrance of Kam Sweet Rice
}

\author{
Zheng Kong ${ }^{1,2}$, Degang Zhao ${ }^{1,2,3, *}$ \\ ${ }^{1}$ Guizhou Key Laboratory of Agro-Bioengineering, Guizhou University, South Campus, Huaxi, Guiyang City, Guizhou Province, \\ People's Republic of China \\ ${ }^{2}$ Key Laboratory of Green Pesticide and Ago-Bioengineering, Ministry of Education, Guizhou University, North Campus, Huaxi, \\ Guiyang City, Guizhou Province, People's Republic of China \\ ${ }^{3}$ College of Life Science, Guizhou University, South Campus, Huaxi, Guiyang City, Guizhou Province, People's Republic of China \\ *Corresponding author: dgzhao@gzu.edu.cn
}

Received June 17, 2014; Revised July 17, 2014; Accepted July 21, 2014

\begin{abstract}
This study aimed to characterize the effect of jasmonic acid (JA) on volatile compounds in grains of Kam sweet rice. Using GC-O and SPME-GC-MS, nonanal displayed the highest odor activity value (OAV) in the filling grains and increased after applicaton of jasmonic acid in the rice seedlings. The relative expression of rice OsLOX3 (rice lipoxygenase 3) and OsHPL1 (rice hydroperoxide lyase 1) was assessed by RT-PCR in Kam sweet rice Gou Cengao and the non-aromatic Kam rice Lailong rice after rice pollination. Our data showed that OsLOX3 was elevated in Gou Cengao compared with the non-aromatic rice. In agreement, lipoxygenase (OsLOX3 gene product) levels and activity were elevated in aromatic rice samples. The positive Pearson correlation (0.715) was found between JA and lipoxygenase activity $(p<0.01)$. Interestingly, a significant positive Pearson correlation (0.936) was found between the concentrations of endogenous JA and the relative expression of rice OsLOX3 ( $p<$ 0.01). The results suggest that the enhancing effect of JA on the biosynthetic pathway of nonanal.
\end{abstract}

Keywords: Kam sweet rice, Nonanal, 2-Acetyl-1-pyrroline, Lipoxygenase activity, Jasmonic acid, OsLOX3

Cite This Article: Zheng Kong, and Degang Zhao, "The Enhancing Effect of Jasmonic Acid on Fragrance of Kam Sweet Rice.” Journal of Food and Nutrition Research, vol. 2, no. 7 (2014): 395-400. doi: 10.12691/jfnr-2-711.

\section{Introduction}

The Asian cultivated rice (Oryza sativa L.) is one of the most important crops and a major food source for more than half of the global human population [1]. Among all sticky rice varieties cultivated worldwide, Kam sweet rice is known for its pleasant fragrance and cultivated for generations without using chemical pesticides or fertilizers [2]. Kam sweet rice is a major type of cultivated rice with a long history and culinary importance in China [3]. Indigenous aromatic Kam sweet rice types cultivated in China include Gou Cengao, Gou Gaoqian and Congjiang, found especially in southwest China where Kam sweet rice is traditional foods. However, major aromatic components found in the grains of Kam sweet rice have not been characterized.

Multiple studies aimed to explore factors that regulate the volatile components from aromatic rice. For example, trace amounts of gibberellic acid result in decreased 2acetyl-1-pyrroline levels in aromatic rice [4], and many aromatic components have been markedly noticed in aromatic rice after treatment with abscisic acid (ABA) [5]. The aroma quality of rice is affected by environmental factors: rice cropped in rain-fed paddy fields shows higher content of aromatic compounds [6]; the yield of aromatic volatile compounds is affected by salinity $[7,8,9]$. In addition, enzyme activity is an important factor in volatile compounds synthesis of aromatic rice. Indeed, 2-acetyl-1pyrroline synthesis was regulated by the expression level of $\Delta^{1}$-pyrroline-5-carboxylixc acid synthetase OsP5CS [10] and it has been shown that volatile aldehydes are regulated by peroxidation and lipid oxidation [11]. Finally, Tomio et al. reported that 2-acetyl-1-pyrroline content in rice was higher when ripened at low temperature (day: $25^{\circ} \mathrm{C} /$ night: $20^{\circ} \mathrm{C}$ ) compared with high temperature (day: $35^{\circ} \mathrm{C} /$ night: $30^{\circ} \mathrm{C}$ ) (Itani et al. 2004). Likewise, variation of temperature was suggested as an alternative way to affect aroma changes [12].

In this study, JA amounts and the volatile components of Kam sweet rice Gou Cengao during filling stage were analyzed to determine the correlation between hormone and nonanal, the key volatile compound.

\section{Materials and Methods}

Plant Materials. The Kam sweet rice (Gou Cengao) and Kam rice (Lailong) are Chinese japonica rice found in the Guizhou Plateau, which is located in southwest China. The samples were milled to remove bran layers, and samples sealed in zip lock bages were kept at $-80^{\circ} \mathrm{C}$ until use. Fifteen-day-old seedlings with three leaves were either treated with water supplemented with $120 \mathrm{mg} / \mathrm{L} \mathrm{JA}$ or water alone for $48 \mathrm{~h}$. The green leaves were harvested 
and samples of equal weight (1.0 g) were homogenized in liquid nitrogen for detetion of volatile components.

SPME Sampling. The equilibration time 20 mins has been enough for most volatile compounds in headspace solid-phase microextraction (SPME). Hence, SPME was carried out by a DVB fiber $(50 / 30 \mu \mathrm{m}, 1 \mathrm{~cm})$ attached to a manual holder from Supelco (Bellefonte, PA, USA). Then, the DVB fiber was desorbed for $5 \mathrm{~min}$ on an Agilent 5975C/6890 GC equipped with a HP 5973 mass-selective detector (Agilent Technologies, Palo Alto, CA, USA) and a $30 \mathrm{~m} \times 0.25 \mathrm{~mm} \times 0.25 \mu \mathrm{m}$ Zebron ZB-5MSI fused silica capillary column (5\% Phenyl-95\% Dimethylpolysiloxane) from Zebron (USA). The injector temperature was kept at $250^{\circ} \mathrm{C}$ throughout the separation.

Gas chromatography-mass spectrometry (GC-MS) and Gas chromatography-olfactometry (GC-O). GCMS experiments were carried out on an Agilent 6890/HP 5973 instrument. Samples were injected in split mode. The column temperature started at $40^{\circ} \mathrm{C}$ with a 1 min hold followed by increase at incremental rates of $4{ }^{\circ} \mathrm{C} / \mathrm{min}$ to $150^{\circ} \mathrm{C}$. The interface temperature was programed at $280^{\circ} \mathrm{C}$ and the flow rate of the carrier gas-helium was 1.0 $\mathrm{mL} / \mathrm{min}$. Volatiles were split between the mass spectrometer and the olfactory detection port (Gersterl ODP2, Germany) for description and intensity assessment. The mass spectrometer was operated in an electron impact (EI) ionization mode with electron energy of $70 \mathrm{eV}$ and electron multiplier voltage of $1052 \mathrm{~V}$. The temperature of the ion source was $230^{\circ} \mathrm{C}$ and mass spectra were obtained by scanning from $\mathrm{m} / \mathrm{z} 20-450$. For olfactory detection, three independent assessors trained for odor description of volatiles evaluated the samples. Odor intensity was classified as nd (not detected), 1 (very weak), 2 (weak), 3 (intermediate), 4 (strong), and 5 (very strong). The volatiles perceived by all assessors were considered odor active components.
Extraction of JA. Samples (1g each) were freeze-dried and ground in $10 \mathrm{~mL}$ of methanol mixture (80\%) in weak light. The homogenates were stored at $4^{\circ} \mathrm{C}$ for $24 \mathrm{~h}$ and submitted to centrifugation $\left(4^{\circ} \mathrm{C}, 5000 \mathrm{r} / \mathrm{m}, 10 \mathrm{~min}\right)$. After methanol removed (vacuum at $35-40^{\circ} \mathrm{C}$ ), the aqueous phase were mixed with $100 \mu \mathrm{L}$ ammonia. Then, samples were filtered on polyvinylpyrrolidone (PVPP) and were mixed with $\mathrm{HCl}$ ( $2 \mathrm{~mol} / \mathrm{L}$ ) to reduce $\mathrm{pH}$ to 3.0. The samples were extracted by equal volume ethyl acetate and mixed with $100 \mu \mathrm{L}$ ammonia. After desiccating with rotary evaporator $\left(40^{\circ} \mathrm{C}\right)$, the samples were dissolved with acetic acid $(0.1 \mathrm{~mol} / \mathrm{L}, 5 \mathrm{~mL})$. The samples collected by Sep-Pak C18 $(1 \mathrm{~cm} \times 15 \mathrm{~cm}, 30 \mathrm{~mL})$ were eluted with $40 \%$ methanol. The resulting samples desiccated with rotary evaporator $\left(40^{\circ} \mathrm{C}\right)$ were dissolved with water: acetonitrile (85: 15, 0.05\% acetic acid) for HPLC analysis.

Identification and Quantification of Volatile compounds and JA. The following standards were used for the identification of aromatic compounds in cooked rice samples: pentanol, hexanal, heptanal, $(E)$-2-hexenal, 1-octen-3-ol, octanal, (E)-2-octenal, octanol, nonanal and decanal were purchased from Aladdin-reagent (China); benzaldehyde was provided by Xiyashiji (China) and 2acetyl-1-pyrroline by J\&K Chemical (China). The nalkanes $\mathrm{C}_{6}-\mathrm{C}_{19}$ (AccuStandard, USA) were used to derive the retention indices. JA was provided by Aladdin-reagent (China).

All aromatic compounds were identified by matching with mass spectra in the NIST and WILEY libraries. Confirmation was carried out by comparing retention times with those authentic standards. For quantification of the compounds, standard curves were generated by analyzing GC-MS data after injection of different concentrations (ppm) of the compounds diluted in hexane (Table 1).

Table 1. Linearity, Sensitivity and Precision of Major Odor-Active Compounds detected in Kam Sweet Rice Sample after pollination

\begin{tabular}{|c|c|c|c|c|}
\hline Compound & Standard Curve & $\mathrm{R}^{2}$ & Validation Range & RSD(\%) \\
\hline pentanol & $y=3 \times 10^{-6} x+0.5893$ & 0.9999 & $0.3-199$ & 1.01 \\
\hline hexanal & $y=9 \times 10^{-6} x+1.3659$ & 0.9997 & $0.3-202$ & 2.50 \\
\hline (E)-2-hexenal & $y=5 \times 10^{-6} x+0.9504$ & 0.9997 & $0.5-199$ & 0.99 \\
\hline heptanal & $y=5 \times 10^{-6} x+0.2959$ & 0.9994 & $0.3-198$ & 3.83 \\
\hline 2-acetyl-1-pyrroline & $y=9 \times 10^{-7} x+0.0402$ & 0.9997 & $0.04-4.9$ & 3.48 \\
\hline benzaldehyde & $y=2 \times 10^{-5} x-0.8293$ & 0.9997 & $0.3-188$ & 0.37 \\
\hline 1-octen-3-ol & $y=9 \times 10^{-6} x+0.5793$ & 0.9999 & $2.9-180$ & 3.19 \\
\hline octanal & $y=6 \times 10^{-6} x-1.8464$ & 0.9993 & $1.0-150$ & 1.91 \\
\hline (E)-2-octenal & $y=5 \times 10^{-6} x+3.7986$ & 0.9997 & $2.9-218$ & 2.31 \\
\hline octanol & $y=5 \times 10^{-6} x+4.3261$ & 0.9996 & 3.3-195 & 2.21 \\
\hline nonanal & $y=1 \times 10^{-5} x+18.384$ & 0.9992 & 24-199 & 0.29 \\
\hline decanal & $y=7 \times 10^{-6} x-0.5105$ & 0.9999 & $1.0-200$ & 2.18 \\
\hline
\end{tabular}

For HPLC, acetonitrile and a mixed solution containing 99.95\% water and $0.05 \%$ acetic acid were used as mobile phase. The flow rate was $300 \mu \mathrm{L} / \mathrm{min}$, with a column maintained at $30^{\circ} \mathrm{C}$. A total of $10 \mu \mathrm{L}$ sample were injected. The gradient elution: $1-3 \mathrm{~min}, 15 \%$ acetonitrile; $3-5 \mathrm{~min}$, 15\%-100\% acetonitrile; 5-6 min, 100\% acetonitrile; 6-7 min $100 \%-15 \%$ acetonitrile, $7-8 \quad \min , \quad 15 \%$ acetonitrile.The equation was obtained for JA standard curve of JA: $y=25342.6 x-1929.14$, with $r^{2}=0.9992$, with a validation range comprised between 3.1 and $102 \mathrm{ng} / \mathrm{g}$ and RSD of 3.32\%.

RNA isolation and semi-quantitative RT-PCR. Total RNA was isolated from rice grain samples obtained from the aromatic Kam sweet rice Gou Cengao and nonaromatic Kam rice Lailong. RNA extraction from ten independent samples was carried out at four distinct stages of the rice grain development $(6,8,10$, and 15 days after rice flowering), using the E.Z.N.A ${ }^{\mathrm{TM}}$ Plant RNA Kit (OMEGA, USA) and following manufacturer's instructions:

1. Collect frozen ground plant tissue(up to $100 \mathrm{mg}$ ) in a microfuge tube and immediately add $500 \mu \mathrm{L}$ Buffer RCL/2-mercaptoethanol. We recommend starting with 50 mg tissues at first. If results obtained are satisfactory increase amount of starting material. Samples should not be allowed to thaw before Buffer RCL/2-mercaptoethanol is added. Vortex vigorously to make sure that all clumps are dispersed. RNA cannot be effectively extracted from clumped tissue.

2. Incubate at $55^{\circ} \mathrm{C}$ for $1-3$ minutes. Centrifuge at maxi speed $(14000 \times \mathrm{g})$ for $5 \mathrm{~min}$ at room temperature. 
3. Transfer the supernatant directly into a gDNA Filter Column in $2 \mathrm{~mL}$ collection tube and centrifuge at $14000 \times$ $\mathrm{g}$ for $2 \mathrm{~min}$ at room temperature.

4. Add eqaul volume Buffer RCB to the flow-through and mix well by pipetting up and down 5-10 times.

5. Apply one half of the mixture from step 4 to a HiBind@ RNA Mini column assembled in a clean $2 \mathrm{~mL}$ collection tube (supplied). Centrifuge at $10000 \times \mathrm{g}$ for 1 min at room temperature. Discard the flow-through liquid and place the column back into the collection tube.

6. Apply the regaining of the mixture from step 4 to the column. Centrifuge at $10000 \times \mathrm{g}$ for $1 \mathrm{~min}$ at room temperature. Discard the flow-through liquid and place the column back into the collection tube.

7. Add $400 \mu \mathrm{L}$ RWC Wash Buffer and centrifuge as above. Discard both flow-through liquid and collection tube.

8. Place column in a clean $2 \mathrm{~mL}$ collection tube (supplied), and add $500 \mu \mathrm{L}$ RNA Wash Buffer II diluted with ethanol. Centrifuge as above and discard flowthrough. Re-use the collection tube in step 9.

9. Wash column with a second $500 \mu \mathrm{L}$ RNA Wash Buffer II by repeating step 8. Centrifuge as above and discard flow-through. Then with the collection tube empty, centrifuge the column for $2 \mathrm{~min}$ at $10000 \times \mathrm{g}$ to completely dry the column matrix.

10. Elution of RNA: Transfer the column to a clean 1.5 $\mathrm{mL}$ microfuge tube (not suplied) and elute the RNA with 30-50 $\mu \mathrm{L}$ of DEPC water (suplied). Make sure to add water directly onto column matrix. Incubate at room temperature for $2 \mathrm{~min}$. Centrifuge for $1 \mathrm{~min}$ at $10000 \times \mathrm{g}$. A second elution into the same tube may be necessary if the expected yield of RNA $>30 \mu \mathrm{g}$.

Reverse transcription was carried out separately for 10 $\min \left(25^{\circ} \mathrm{C}\right), 120 \mathrm{~min}\left(37^{\circ} \mathrm{C}\right)$ and $5 \mathrm{~min}\left(85^{\circ} \mathrm{C}\right)$, using the high-capacity cDNA reverse transcription kit (Applied Biosystems, USA). To prepare the $2 \times$ RT master mix on ice (per $20 \mu \mathrm{L}$ reaction): $10 \times$ RT Buffer $2.0 \mu \mathrm{L} ; 25 \times$ dNTP Mix (100 mM) $0.8 \mu \mathrm{L} ; 10 \times$ RT Random Primers $2.0 \mu \mathrm{L}$; Multiscribe ${ }^{\mathrm{TM}}$ Reverst Transcriptase $1.0 \mu \mathrm{L}$; Rnase Inhibitor $1.0 \mu \mathrm{L}$; Nuclease-free $\mathrm{H}_{2} \mathrm{O} 3.2 \mu \mathrm{L}$. To prepare the cDNA Reverse Transcription reactions: 1. Pipette $10 \mu \mathrm{L}$ of $2 \times$ RT master mix into each well of a 96well reaction plate or individual tube. 2. Pipette $10 \mu \mathrm{L}$ of RNA sample into each well, pipetting up and down two times to mix. 3. Seal the plates or tubes. 4. Briefly centrifuge the plate or tubes to spin down the contents and to eliminate any air bubbles. 5. Place the plate or tubes on ice until you are ready to load the thermal cycler.

Real-Time PCR was carried out on an ABI 7500 Fast Real Time PCR instrument with the 7500 v2.0.1 software (Applied Biosystems, USA). RT-PCR conditions included an initial denaturation at $95^{\circ} \mathrm{C}$ for $10 \mathrm{~min}$ followed by 40 cycles of $95^{\circ} \mathrm{C}$ for $15 \mathrm{~s}$ and $60^{\circ} \mathrm{C}$ for $1 \mathrm{~min}$. The melting curves were obtained at $95^{\circ} \mathrm{C}$ for $15 \mathrm{~s}, 60^{\circ} \mathrm{C}$ for $1 \mathrm{~min}$; and $95^{\circ} \mathrm{C}$ for $15 \mathrm{~s}$. The following primers were used: OsLOX3 Forward, 5'-CAACAGGCTCTACATTCT-3'; OsLOX3 Reverse, 5'-GTGGCATAGGTGAAGATA-3' (Reference sequence: GenBank FJ660622.1); OsHPL1 Forward, 5'AGCTCCTCCACAACCTCG-3'; OsHPL1 Reverse, 5'CGATGTGTGGCAGGAAGAT-3' (Reference sequence: GenBank AK105964.1). The ubiquitin-conjugating enzyme E2 gene was employed as internal control:
Forward primer 5'CCGTTTGTAGAGCCATAATTGCA-3'; Reverse primer 5'-AGGTTGCCTGAGTCACAGTTAAGTG-3'

(Reference sequence: GenBank AK059694.1). mRNA levels were expressed relatively to the ubiquitinconjugating enzyme E2 gene in each sample.

Lipoxygenase Extraction and Enzyme Activity. Lipoxygenase was extracted as previously described [10]. Rice grains were frozen and ground in liquid nitrogen. The resulting powder was stored at $-20^{\circ} \mathrm{C}$ until use. For extraction, $1 \mathrm{~g}$ powder was added to $10 \mathrm{~mL}$ phosphate buffer (1\% PVP, pH 6.5, $0.2 \mathrm{M}$ ) and the suspension centrifuged at $10000 \times \mathrm{g}$ for $20 \mathrm{~min}\left(4^{\circ} \mathrm{C}\right)$. Ammonium sulfate was added to supernatants to achieve 35\% saturation and the mixture was centrifuged as described above. The resulting supernatants were precipitated with $70 \%\left(\mathrm{NH}_{4}\right)_{2} \mathrm{SO}_{4}$ for $1 \mathrm{~h}$ and the samples submitted to centrifugation $\left(4^{\circ} \mathrm{C}, 10000 \times \mathrm{g}, 20 \mathrm{~min}\right)$. The pellet containing the enzyme was dissolved in $2 \mathrm{~mL}$ of $0.01 \mathrm{M}$ phosphate buffer (pH6 .5) containing 10\% glycerol. The samples were desalinated by Sephadex G-25 column (1 $\mathrm{cm} \times 15 \mathrm{~cm}, 30 \mathrm{~mL}$ ) and stored at $4^{\circ} \mathrm{C}$. Enzyme activity was determined as previously described [13]. Briefly, quartz color dishes were filled with $0.2 \mathrm{~mL}$ substrate, 2.75 $\mathrm{mL}$ phosphate buffer ( $\mathrm{pH}$ 6.5, $0.2 \mathrm{M})$ and $50 \mu \mathrm{L}$ lipoxygenase extract and activity was assessed spectrophotometrically by monitoring the formation of conjugated dienes at $234 \mathrm{~nm}$. The enzyme activity unit was calculated as $\Delta \mathrm{A}_{234} \mathrm{~min}^{-1} \mathrm{~g}^{-1} \mathrm{FW}$. The substrate solution was prepared as proposed by Engeseth et al. [14]. Briefly, $0.1 \mathrm{~mL}$ linoleic acid stock solution was mixed with $2 \mathrm{~mL}$ boric acid (pH 9.0, 0.2 M) and 0.1 mL Tween20. Then, $0.2 \mathrm{~mL} \mathrm{NaOH}(1 \mathrm{M})$ was added and mixed until a clear solution was obtained. Finally, boric acid was added to increase the solution volume to $40 \mathrm{~mL}$. The substrate solution was stored at $20^{\circ} \mathrm{C}$ until use.

Statistical Analysis. Data were analyzed by SPSS version 16.0 (SPSS Inc., Chicago, Il) and Pearson correlation. The Duncan's multiple-range test was used to compare OAVs of the odor-active compounds from rice samples.

\section{Results and Discussion}

The relative intensities and descriptors of odor-active compounds emanating from aromatic Kam sweet rice Gou Cengao rice after pollination, as determined by trained assessors, are summarized in Table 2. Aldehydes constituted the most abundant group among the 12 odoractive compounds detected in Kam sweet rice Gou Cengao. Indeed, 7 aldehydes were found, including hexanal, (E)-2-hexenal, heptanal, octanal, $(E)$-2-octenal, nonanal and decanal. The remaining constituents is a aromatic compound (benzaldehyde), two alcohols (pentanol, 1-octen-3-ol and 1-octanol) and a nitrogencontaining compound (2-acetyl-1-pyrroline). Buttery et al. [15] reported 2-acetyl-1-pyrroline and $(E, E)-2$, 4decadienal as key aroma components in cooked rice, although these compounds were not found cooked Gou Cengao as shown above. This is likely due to the difference in the developmental stages of rice studied. Based on odor intensity, nonanal contributed most to the flavor of Gou Cengao, with odor intensity values after 
pollination of 1.6 at sixth day, 2.1 at eighty day. and 2.4 at fifteenth day after, respectively. The popcorn-like 2- acetyl-1-pyrroline contributed the most odor intensity (2.1) at tenth day after pollination.

Table 2. Odor Intensity and Description of Odor-Active Compounds in Kam Sweet Rice after pollination:6 days, 8 days, 10 days, 15 days

\begin{tabular}{|c|c|c|c|c|c|c|c|}
\hline \multirow{2}{*}{$\mathrm{RI}^{\mathrm{b}}$} & \multirow{2}{*}{ Odorant } & \multicolumn{4}{|c|}{ Odor intensity $^{\mathrm{a}}$} & \multirow{2}{*}{ Odor description $^{c}$} & \multirow{2}{*}{ Identification $^{\mathrm{d}}$} \\
\hline & & 6 days & 8 days & 10 days & 15 days & & \\
\hline 627 & pentanol & nd & 1.0 & nd & nd & plastic & MS,STD \\
\hline 643 & hexanal & 1.2 & 1.3 & 1.2 & 1.2 & green & MS.STD,RI \\
\hline 711 & (E)-2-hexenal & nd & 1.2 & 1 & nd & green & MS,STD,RI \\
\hline 744 & heptanal & 1.4 & 1.7 & 1.1 & 1.1 & floral & MS,STD,RI \\
\hline 793 & 2-acetyl-1-pyrroline & nd & 1.6 & 2.1 & 1.4 & popcorn & MS,STD,RI \\
\hline 826 & benzaldehyde & nd & 1.3 & nd & nd & almond & MS,STD,RI \\
\hline 849 & 1-octen-3-ol & nd & 1.0 & nd & nd & mushroom & MS,STD,RI \\
\hline 869 & octanal & 1.1 & 1.7 & 1.3 & 1.4 & green citrus & MS,STD,RI \\
\hline 906 & (E)-2-octenal & nd & 1.3 & nd & nd & nutty & MS,STD,RI \\
\hline 920 & octanol & nd & 1.4 & 1.2 & 1.1 & citrus & MS,STD,RI \\
\hline 968 & nonanal & 1.6 & 2.1 & 1.9 & 2.4 & citrus fatty & MS,STD,RI \\
\hline 1041 & decanal & 1.1 & 1.0 & 1.2 & 1.3 & citrus & MS,STD,RI \\
\hline
\end{tabular}

${ }^{a}$ Average intensity of compounds that were detected by all three assessors.nd=not detected. ${ }^{b}$ Retention indexs were counted using a series of n-alkanes $\mathrm{C}_{6}-\mathrm{C}_{19 .}{ }^{c}$ Odorants were described by assessors during GC-O. ${ }^{d}$ MS, by comparison of the mass spectrum with the NIST/Wiley mass spectral library; RI, by comparison of RI with those from the literature;STD, by comparison of retention time, spectrum, and odor description of an identified compound with those of an authentic compound.

To assess the contribution of individual odor active component to the overall aroma, the compounds detected by the assessors (i.e., odor intensity $>0$ ) were considered potentially contributors [16,17]. OAVs, obtained by dividing each component's concentration by its odor threshold in the air, were used to assess the relative importance of individual aromatic compounds in rice aroma (Table 3.). To determine the concentrations of the odor-active components, excellent standard curves were established using authentic commercially available standards, with linear correlation coefficients $\left(\mathrm{R}^{2}\right)$ ranging from 0.9992 to 0.9999 and relative standard deviations (RSDs) varying from 0.29 to $3.83 \%$ (Table 1.). As shown in Table 3., nonanal was the most potent odor-active compound in the Kam sweet rice Gou Cengao within 15 days after pollination $(\mathrm{OAV}=12.6,20.4,15.2,16.0$; relative proportion $=49.8 \%, 32.4 \%, 33.4 \%, \quad 50.1 \%$ ), followed by 2-acetyl-1-pyrroline (OAV=0, 7.7, 19.8, 7.2; relative proportion $=0 \%, 12.2 \%, 43.5 \%, 22.5 \%$ ), octanal $(\mathrm{OAV}=7.1,10.4,3.8,3.9$; relative proportion $=27.4 \%$, 16.5\%, 8.4\%, 12.2\%), hexanal (OAV=3.8, 13.5, 3.2, 2.1; relative proportion=14.6\%, 21.5\%, 7.0\%, 6.6\%), heptanal $(\mathrm{OAV}=1.8,3.5,1.6,0.8$; relative proportion $=6.9 \%$, 5.6\%, $3.5 \%, 2.5 \%)$, decanal $(\mathrm{OAV}=0.6,1.7,0.5,1.7$; relative proportion=2.3\%, 2.7\%, 1.1\%, 5.3\%), (E)-2-octenal $(\mathrm{OAV}=0,1.8,0,0$; relative proportion $=0 \%, 2.8 \%, 0 \%$, $0 \%)$ (E)-2-hexenal $(\mathrm{OAV}=0, \quad 1.4, \quad 0.9, \quad 0$; relative proportion=0\%, 2.2\%, 2.0\%, $0 \%$ ), and 1-octen-3-ol $(\mathrm{OAV}=0,1.4,0,0$; relative proportion $=0 \%, 2.2 \%, 0 \%, 0 \%)$.

Aldehydes, especially nonanal, constituted the greast odor active contributors of the aromatic Kam sweet rice Gou Cengao. Interestingly, C9-aldehydes are significant odorants in many rice types and known products of 9lipoxygenase and 9-hydroperoxide lyase, which are encoded separately by OsLOX3 [18] and OsHPL1 [19] gene, respectively.

Table 3. Odor Activity Values (OAVs) of Major Odor-Active Compounds in Kam Sweet Rice after pollination:6 days,8 days,10 days,15 days

\begin{tabular}{|c|c|c|c|c|c|c|}
\hline \multirow[b]{2}{*}{$R I^{b}$} & \multirow[b]{2}{*}{ odorant } & \multicolumn{4}{|c|}{$\mathrm{OAV}^{\mathrm{a}}(\mathrm{n}=3)$} & \multirow[b]{2}{*}{ odor threshold in air(ng/L) } \\
\hline & & 6 days & 8 days & 10 days & 15 days & \\
\hline 627 & pentanol & nd & $0.02 \pm 0.0003 a$ & nd & nd & $153^{c}$ \\
\hline 643 & hexanal & $3.8 \pm 0.08 b$ & $13.5 \pm 0.17 \mathrm{a}$ & $3.2 \pm 0.01 \mathrm{c}$ & $2.1 \pm 0.004 \mathrm{~d}$ & $1.1^{c}$ \\
\hline 711 & (E)-2-hexenal & nd & $1.4 \pm 0.01 \mathrm{a}$ & $0.9 \pm 0.004 \mathrm{~b}$ & nd & $3.1^{c}$ \\
\hline 744 & heptanal & $1.8 \pm 0.03 \mathrm{~b}$ & $3.5 \pm 0.02 \mathrm{a}$ & $1.6 \pm 0.01 \mathrm{c}$ & $0.8 \pm 0.002 \mathrm{~d}$ & $0.9^{c}$ \\
\hline 793 & 2-acetyl-1-pyrroline & nd & $7.7 \pm 0.15 b$ & $19.8 \pm 0.21 \mathrm{a}$ & $7.2 \pm 0.06 \mathrm{c}$ & $0.02^{d}$ \\
\hline 826 & benzaldehyde & nd & $0.6 \pm 0.001 \mathrm{a}$ & nd & nd & $85^{c}$ \\
\hline 849 & 1-octen-3-ol & nd & $1.4 \pm 0.01 \mathrm{a}$ & nd & nd & $2.7^{c}$ \\
\hline 869 & octanal & $7.1 \pm 0.13 b$ & $10.4 \pm 0.08 \mathrm{a}$ & $3.8 \pm 0.06 \mathrm{c}$ & $3.9 \pm 0.04 \mathrm{c}$ & $0.4^{c}$ \\
\hline 906 & (E)-2-octenal & nd & $1.8 \pm 0.006 \mathrm{a}$ & nd & nd & 2.7 \\
\hline 920 & octanol & nd & $0.4 \pm 0.002 \mathrm{~b}$ & $0.5 \pm 0.0009 a$ & $0.2 \pm 0.0003 c$ & $22^{c}$ \\
\hline 968 & nonanal & $12.6 \pm 0.02 \mathrm{~d}$ & $20.4 \pm 0.13 a$ & $15.2 \pm 0.005 \mathrm{c}$ & $16.0 \pm 0.01 \mathrm{~b}$ & $2.6^{c}$ \\
\hline 1041 & decanal & $0.6 \pm 0.007 \mathrm{~b}$ & $1.7 \pm 0.007 \mathrm{a}$ & $0.5 \pm 0.008 \mathrm{c}$ & $1.7 \pm 0.02 \mathrm{a}$ & $2.6^{c}$ \\
\hline
\end{tabular}

${ }^{a} \mathrm{OAV}$ is obtained by dividing the concentration of an odor-active compound by its odor threshold in air and means of three replicates;means separation within rows by Duncan's multiple-range test $P<0.05 .{ }^{b}$ Retention indexs were counted using a series of n-alkanes $\mathrm{C}_{6}$ - $\mathrm{C}_{19}{ }^{c}{ }^{c}$ Data from Yang [16]. ${ }^{d}$ Data from Schieberle [17].

Therefore, to understand the highest OAV of nonanal, we assessed the changes in lipoxygenase activity (Table 4.), relative gene expression of OsLOX3 and OsHPL1 (Table 5.) and JA concentrations (Table 5.) in grains 6, 8, 10 , and 15 days after pollination of Kam sweet rice Gou Cengao. As shown in Table 2, nonanal was found at all stages of the grain development in Kam sweet rice Gou Cengao. Interestingly, OsLOX3 expression was significantly higher in Gou Cengao than in Lailong after pollination. However, no constant trend was found for OsHPL1 expression, which was lower in Gou Cengao

compared with Lailong at 6 (1.935 vs. 17.143), 8 (14.713 vs. 18.209$)$ and 15 days (0.1.5 vs. 0.982$)$ but slightly higher at 10 days (14.675 vs. 12.889). Therefore, OsLOX3 was considered the most critical gene in Gou Cengao. To confirm these findings, enzyme activity of lipoxygenase was assessed in the same samples (Table 4.). In agreement with gene expression data, lipoxygenase activity was markedly increased at all time points during rice filling. Finally, using Pearson correlation analysis, we found that lipoxygenase activity correlated with JA amounts with a positive correlation of $r=0.751$ (two-tailed analysis, $p<$ 
0.01) and OAV variation in nonanal amounts (6 days vs. 8 days; 8 days vs. 10 days; 10 days vs. 15 days) correlated with JA amounts, with a significant positive correlation of $r=0.981$ (two-tailed analysis, $p<0.01$ ). Interestingly the variation in JA amounts correlated with the OsLOX3 expression, with a significant positive correlation of $r=$ 0.936 (two-tailed analysis, $p<0.01$ ) (Table 5.). Using Pearson correlation analysis, we found no significant correlation between JA concentration and OsHPL1 expression (correlation of $r=0.045$, two-tailed analysis, $p>0.05$ ) (Table 5.). These results suggested that OsLOX3 was also the most critical gene in JA regulation pathway of nonanal. Importantly, we found that JA had enhancing effects on nonanal biosynthesis. To confirm these findings, nonanal concentrations were assessed in the rice seedlings after application of JA. In agreement with the positive correlation described above between $\mathrm{JA}$ and OsLOX3 expression, nonanal amounts were significantly higher in Gou Cengao seedlings treated with JA than controls (86.238 vs. 54.519) (Table 6.).

Table 4. The enzyme activity of lipoxygenase in Kam Sweet Rice after pollination:6 days,8 days,10 days,15 days

\begin{tabular}{ccc}
\hline Time & \multicolumn{2}{c}{ enzyme activity of lipoxygenase $\left(\Delta \mathrm{A}_{234} \mathrm{~min}^{-1} \mathrm{~g}^{-1} \mathrm{FW}\right)$} \\
\hline 6day & aromatic Gou Cengao & $0.049 \pm 0.010$ \\
8day & $0.246 \pm 0.047$ & $0.039 \pm 0.0009$ \\
10day & $0.229 \pm 0.038$ & $0.018 \pm 0.004$ \\
15day & $0.266 \pm 0.031$ & $0.006 \pm 0.001$ \\
\hline
\end{tabular}

${ }^{a}$ Time, sampling after rice pollination.

Table 5. The quatification of JA and the relative expression value of OsLOX3 and OsHPL1 in Kam Sweet Rice after pollination: 6 days, 8 days, 10 days, 15 days

\begin{tabular}{ccccc}
\hline \multirow{2}{*}{ Time $^{a}$} & \multirow{2}{*}{ quatification of JA (ng/g) } & \multicolumn{2}{c}{ relative expression value of OsLOX3 } & \multicolumn{2}{c}{ relative expression value of OsHPL1 } \\
& & aromatic Gou Cengao & Lailong as control & aromatic Gou Cengao \\
\hline 6day & $19.480 \pm 1.456$ & $4.625 \pm 0.059$ & $0.931 \pm 0.019$ & $1.935 \pm 0.078$ \\
8day & $9.398 \pm 0.529$ & $2.043 \pm 0.006$ & $0.358 \pm 0.047$ & $14.713 \pm 0.112$ \\
10day & $14.681 \pm 0.554$ & $4.617 \pm 0.277$ & $0.614 \pm 0.020$ & $14.143 \pm 1.624$ \\
15day & $5.522 \pm 0.311$ & $0.633 \pm 0.034$ & $0.045 \pm 0.001$ & $12.875 \pm 0.356$ \\
\hline
\end{tabular}

${ }^{a}$ Time, sampling after rice pollination.

Table 6. The quatification of nonanal in air from the leaves of Kam Sweet Rice seedlings during JA (120mg/L, 48h) treatment

\begin{tabular}{cccccc}
\hline $\mathrm{RI}^{\mathrm{a}}$ & Odorant & JA-treated seedlings (ng/L) & Control (ng/L) & Odor description $^{\mathrm{b}}$ \\
\hline 968 & nonanal & $86.238 \pm 0.901$ & $54.519 \pm 2.523$ & Identification $^{\mathrm{c}}$ & green fatty \\
\hline
\end{tabular}

${ }^{a}$ Retention indexs were counted using a series of n-alkanes $\mathrm{C}_{6}-\mathrm{C}_{19} \cdot{ }^{b}$ Odorants were described by assessors during GC-O. ${ }^{c}$ MS, by comparison of the mass spectrum with the NIST/Wiley mass spectral library;RI, by comparison of RI with those from the literature;STD, by comparison of retention time, spectrum, and odor description of an identified compound with those of an authentic compound.

\section{Conclusions}

In this study, the aroma profile of Kam sweet rice Gou Cengao was determined by a combination of GC-O and GC-MS. Nonanal and 2-acetyl-1-pyrroline were considered the most potent flavor compounds, with nonanal and 2-acetyl-1-pyrroline displaying the highest OAV at different stages after pollination. In addition, OsLOX3 gene was expressed in relatively higher levels in aromatic rice and this translated into high lipogenase activity. The variation of $O s L O X 3$ expression also leaded to the decrease observed for the OAV of nonanal after 8 day. A positive correlation was also found between nonanal concentrations and lipoxygenase activity, suggesting that the high nonanal OAV observed might results from elevated lipoxygenase activity in Gou Cengao rice. The enhancing effect of JA on nonanal biosynthesis was confirmed after application of JA. Indeed, a positive correlation between the endogenous JA and OsLOX3 gene expression. The findings described herein are different with the past finding that ABA decreased the aroma content of aromatic rice in ABA-treated leaves of rice in our past study [20]. The discrepancy might be due to the different the effects of JA and ABA on the metabolic pathways of nonanal and 2-acetyl-1-pyrroline. Our results suggested that rice of different aromatic compounds might have various flavors according to the hormonal treatment administered.

\section{Acknowledgments}

The authors are grateful to the National Transgenic Major Project of China (2011ZX08010-003) and the Province Science Project in Guizhou (Z [2012] 4008) for their financial support.

\section{Reference}

[1] Choudhury B, Khan ML, Dayanandan S (2013) Genetic structure and diversity of indigenous rice (Oryza sativa) varieties in the Eastern Himalayan region of Northeast India. Springer Plus 2: 1-10.

[2] Stone R (2008) Chinese Province Crafts Pioneering Law to Thwart Biopiracy. Science 320: 732-733.

[3] Olsen KM, Purugganan MD (2002) Molecular evidence on the origin and evolution of glutinous rice. Genetics 162: 941-950.

[4] Piebiep G, Sugunya W, Xiangru T (2011) Decrease in rice aroma after application of growth regulators. Agron 31: 349-359.

[5] Roychoudhury A, Basu S, Sengupta DN (2009) Effects of exogenous abscisic acid on some physiological responses in a popular aromatic indica rice compared with those from two traditional non-aromatic indica rice cultivars. Acta Physiologiae Plantarum 31: 915-926.

[6] Yoshihashi T, Nguyen TTH, Kabaki N (2004) Area dependency of 2-acetyl-1-pyrroline content in an aromatic rice variety, Khao Dawk Mali 105. Japan Agricultural Research Quarterly 38: 105110.

[7] Gay F, Maraval I, Roques S, Gunata Z, Boulanger R, Audebert A, Mestres C (2010) Effect of salinity on yield and 2-acetyl-1pyrroline content in the grains of three fragrant rice cultivars 
(Oryza sativa L.) in Camargue (France). Field crops research 117: 154-160.

[8] Fitzgerald TL, Waters DLE, Brooks LO, Henry RJ (2010) Fragrance in rice (Oryza sativa) is associated with reduced yield under salt treatment. Environmental and Experimental Botany 68: 292-300.

[9] Poonlaphdecha J, Maraval I, Roques S, Audebert A, Boulanger R, Bry X, Gunata Z (2012) Effect of Timing and Duration of Salt Treatment during Growth of a Fragrant Rice Variety on Yield and 2-Acetyl-1-pyrroline, Proline, and GABA Levels. Journal of agricultural and food chemistry 60: 3824-3830.

[10] Huang TC, Teng CS, Chang JL, Chuang HS, Ho CT, Wu ML (2008) Biosynthetic mechanism of 2-acetyl-1-pyrroline and its relationship with $\Delta^{1}$-pyrroline-5-carboxylic acid and methylglyoxal in aromatic rice (Oryza sativa L.) callus. Journal of agricultural and food chemistry 56: 7399-7404.

[11] Ratchatachaiyos K, Theerakulkait C (2009) Isolation and some characteristics of Lipoxygenase from aromatic brown rice (Oryza sativa L.) cv. Khao Dawk Mali 105. Journal of immunoassay \& immunochemistry 30: 106-117.

[12] Itani T, Tamaki M, Hayata Y, Fushimi T, Hashizume K (2004) Variation of 2-acetyl-1-pyrroline concentration in aromatic rice grains collected in the same region in Japan and factors affecting its concentration. Plant production science 7: 178-183.
[13] Suzuki Y, Matsukura U (1997) Lipoxygenase activity in maturing and germinating rice seeds with and without lipoxygenase- 3 in mature seeds. Plant Science 125: 119-126.

[14] Engeseth N, Klein B, Warner K (1987) Lipoxygenase lsoenzymes in Soybeans: Effects on Crude Oil Quality. Journal of Food Science 52: 1015-1019.

[15] Buttery RG, Turnbaugh JG, Ling LC (1988) Contribution of volatiles to rice aroma. Journal of agricultural and food chemistry 36: 1006-1009.

[16] Yang DS, Shewfelt RL, Lee K-S, Kays SJ (2008) Comparison of odor-active compounds from six distinctly different rice flavor types. Journal of agricultural and food chemistry 56: 2780-2787.

[17] Schieberle P (1991) Primary odorants in popcorn. Journal of agricultural and food chemistry 39: 1141-1144.

[18] Marla SS, Singh VK (2012) LOX genes in blast fungus (Magnaporthe grisea) resistance in rice. Functional \& integrative genomics 12: 265-275.

[19] Chehab EW, Raman G, Walley JW, Perea JV, Banu G, Theg S, Dehesh K (2006) Rice HYDROPEROXIDE LYASES with unique expression patterns generate distinct aldehyde signatures in Arabidopsis. Plant physiology 141: 121-134.

[20] Kong Z and Zhao DG (2014) The Inhibiting Effect of Abscisic Acid on Fragrance of Kam Sweet Rice.Journal of Food and Nutrition Research 2: 148-154. 